J. theor. Biol. (1990) 146, 407-432

\title{
Chaotic Dynamics in an Ionic Model of the Propagated Cardiac Action Potential
}

\author{
Timothy J. Lewist市 AND Michael R. Guevara \\ Department of Physiology and Centre for Nonlinear Dynamics in Physiology \\ and Medicine, McGill University, Montreal, Quebec, Canada H3G1Y6
}

\section{(Received and accepted on 20 June 1990)}

\begin{abstract}
We simulate the effect of periodic stimulation on a strand of ventricular muscle by numerically integrating the one-dimensional cable equation using the Beeler-Reuter model to represent the transmembrane currents. As stimulation frequency is increased, the rhythms of synchronization $\{1: 1 \rightarrow 2: 2 \rightarrow 2: 1 \rightarrow 4: 2 \rightarrow$ irregular $\rightarrow 3: 1 \rightarrow$ $6: 2 \rightarrow$ irregular $\rightarrow 4: 1 \rightarrow 8: 2 \rightarrow \ldots \rightarrow 1: 0\}$ are successively encountered. We show that this sequence of rhythms can be accounted for by considering the response of the strand to premature stimulation. This involves deriving a one-dimensional finitedifference equation or "map" from the response to premature stimulation, and then iterating this map to predict the response to periodic stimulation. There is good quantitative agreement between the results of iteration of the map and the results of the numerical integration of the cable equation. Calculation of the Lyapunov exponent of the map yields a positive value, indicating sensitive dependence on initial conditions ("chaos"), at stimulation frequencies where irregular rhythms are seen in the corresponding numerical cable simulations. The chaotic dynamics occurs via a previously undescribed route, following two period-doubling bifurcations. Bistability (the presence of two different synchronization rhythms at a fixed stimulation frequency) is present both in the simulations and the map. Thus, we have been able to directly reduce consideration of the dynamics of a partial differential equation (which is of infinite dimension) to that of a one-dimensional map, incidentally demonstrating that concepts from the field of non-linear dynamics-such as perioddoubling bifurcations, bistability, and chaotic dynamics-can account for the phenomena seen in numerical simulations of the cable equation. Finally, we sketch out how the one-dimensional description can be extended, and point out some implications of our work for the generation of malignant ventricular arrhythmias.
\end{abstract}

\section{Introduction}

Ventricular muscle, which does not beat spontaneously, is normally driven by a periodic electrical input originating in the sinoatrial node of the heart. Periodic stimulation applied at a high rate can lead to a variety of periodic and aperiodic rhythms, including alternans (a rhythm in which there is a beat-to-beat alternation in one or more action potential parameters) and ventricular fibrillation (Savino \&

† Presented by T. J. L. at the "Theory of Hear" workshop held at the University of California at San Diego (La Jolla) in July, 1989.

$\ddagger$ Reprint requests to: T. Lewis, Department of Physiology, McGill University, 3655 Drummond St., Montreal, Quebec, Canada H3G 1 Y6. 
Valentinuzzi, 1988). Within the last decade, the identification of the onset of different forms of alternans with a period-doubling bifurcation has been made in several different cardiac preparations (Guevara et al., 1981, 1984, 1989, 1990; Adam et al., 1982, 1984; Goldberger et al., 1984; Guevara, 1984; Chialvo \& Jalife, 1987; Smith et al., 1988; Hescheler \& Speicher, 1989; Savino et al., 1989; Chialvo et al., 1990). In many of these reports, the alternans is often seen just prior to the phase of induction of irregular rhythms, including ventricular fibrillation. Since it is wellknown that a cascade of period-doubling bifurcations can lead to chaotic dynamics (May, 1976; Thompson \& Stewart, 1986), the hypothesis has been put forth that irregular rhythms, such as fibrillation, might be manifestations of chaotic dynamics occurring as the result of a cascade of period-doubling bifurcations (Guevara et al., 1981; Guevara \& Glass, 1982; Adam et al., 1982; Chialvo \& Jalife, 1987; Smith et al., 1988; Savino et al., 1989). We therefore decided to carry out a systematic study of the effect of pacing at different rates in an ionic model of a strand of ventricular muscle. Our goal was to determine whether or not chaotic dynamics could be produced, and if so, to study the bifurcation sequence leading to chaos.

\section{Cable Simulations}

The transmembrane potential along a $6.25 \mathrm{~mm}$ long cylindrical strand of ventricular muscle was calculated (Sharp \& Joyner, 1980; Victorri et al., 1984; Guevara, 1988), assuming that the strand obeyed the one-dimensional cable equation:

$$
\left(a / 2 R_{i}\right) \partial^{2} V / \partial x^{2}=C \partial V / \partial t+I_{m},
$$

with $x$ the distance along the cable $(\mathrm{cm}), t$ the time $(\mathrm{msec}), V$ the transmembrane potential $(\mathrm{mV}), a$ the cell radius $(7 \cdot 5 \mu \mathrm{m}), R_{i}$ the internal resistivity $(200 \Omega-\mathrm{cm}), C$ the capacitance $\left(1 \mu \mathrm{F} \mathrm{cm}^{-2}\right)$, and $I_{m}$ the net membrane ionic current as described in the Beeler-Reuter ionic model of ventricular muscle (Beeler \& Reuter, 1977). This current is the sum of six individual currents: the fast inward sodium current $\left(I_{\mathrm{Na}}\right)$, the slow calcium current $\left(I_{s}\right)$, the delayed rectifying potassium current $\left(I_{x 1}\right)$, and two background or time-independent currents $\left(I_{K 1}, I_{\mathrm{Na}, C}\right)$. Sealed-end (Dirichlet) boundary conditions were set, and the strand divided into 100 segments, each having a length $(\Delta x)$ of $62.5 \mu \mathrm{m}$. Numerical integration was carried out on a CRAY X-MP computer (14 significant decimal digits), using a mixed implicit-explicit half-step integration routine (Hines, 1984) with a variable time-step $(\Delta t)$ algorithm (Victorri et al., 1985). With $1 \mu \mathrm{sec} \leq \Delta t \leq 8.192 \mathrm{msec}$, the change in transmembrane potential $\Delta V$ from time $t$ to time $t+\Delta t$ was always less than $0.4 \mathrm{mV}$ in all segments. When $\Delta V$ was less than $0.2 \mathrm{mV}, \Delta t$ was doubled for the next iteration. The strand was periodically stimulated by injecting depolarizing current pulses of amplitude $100 \mu \mathrm{A} \mathrm{cm}^{-2}$ and duration $10 \mathrm{msec}$ into its first segment. The first stimulus pulse was always delivered at $t=50 \mathrm{msec}$. The interval between successive stimuli during periodic stimulation is denoted by $t_{s}$. To reduce the length of transients, unless stated otherwise, initial conditions at $t=0$ in all segments were set to values taken from computations employing an isopotential patch of Beeler-Reuter membrane 
stimulated at $t_{s}=500 \mathrm{msec}: V=-83.42 \mathrm{mV}, m=0.01275, h=0.9821, j=0.9683$, $d=0.003280, f=0.9973, x_{1}=0.1533,\left[\mathrm{Ca}^{2+}\right]_{i}=0.1860 \mathrm{mM}$.

With the above parameter values, the surface-to-volume ratio of the strand $\left(2667 \mathrm{~cm}^{-1}\right)$ and the space constant $(1.07 \mathrm{~mm})$ are close to the experimentally accepted values for healthy ventricular muscle, as are the computed conduction velocity $\left(66 \mathrm{~cm} \mathrm{sec}^{-1}\right)$ and the action potential upstroke velocity $\left(99 \mathrm{~V} \mathrm{sec}^{-1}\right)$ in the middle of the cable at $t_{s}=500 \mathrm{msec}$. Thus, the small discretization factor $(\Delta x / \mathrm{space}$ constant) of 0.06 , together with the upper bound of $0.4 \mathrm{mV}$ on $\Delta V$, leads to a relatively low numerical error (Victorri et al., 1984).

We investigate the effect of changing $t_{s}$ in $1 \mathrm{msec}$ decrements. At each $t_{s}$, eqn (1) was integrated to produce a time series at least $10 \mathrm{sec}$ long. When $t_{s}$ is sufficiently large, each stimulus provokes an action potential in the proximal end of the strand, which successfully propagates to its distal end, thus resulting in a $1: 1$ rhythm [Fig. 1(a)]. An $\mathrm{N}: \mathrm{M}$ rhythm $(\mathrm{N} \geq 1, \mathrm{M} \geq 0)$ is periodic with period $N t_{s}$, consisting of repeating $N: M$ cycles, each containing $N$ stimulus pulses and $M$ action potentials with distinct morphologies. As $t_{s}$ is decreased, the $1: 1$ response is maintained down to $t_{s}=307 \mathrm{msec}$, with the action potential duration in the steady-state decreasing. A transient alternation in action potential morphology is increasingly apparent as $t_{s}$ decreases, with both the degree of the alternation and the duration of the transient increasing.

At $t_{s}=306 \mathrm{msec}$, the $1: 1$ rhythm is replaced by a $2: 2$ rhythm. In a $2: 2$ rhythm, there is still one propagated action potential produced by each stimulus, but there is a strict beat-to-beat alternation in the morphology of the action potential and its conduction velocity [Fig. 1(b)]. As $t_{s}$ is lowered further, the degree of alternation in the steady-state becomes more marked. At $t_{s}=293 \mathrm{msec}$, there is an abrupt transition to a $2: 1$ rhythm, in which every second stimulus is blocked, i.e. does not produce a propagated action potential [Fig. 1(c)]. Further decrease in $t_{s}$ to $151 \mathrm{msec}$ leads to a $4: 2$ rhythm, in which every second response is still blocked, as in the $2: 1$ rhythm, but again with an alternation in the morphology of the propagated action potential [Fig. 1(d)]. For $150 \mathrm{msec} \geq t_{s} \geq 140 \mathrm{msec}$, periodic rhythms are no longer seen: only irregular, apparently aperiodic rhythms occur [Fig. 1(e)]. These aperiodic rhythms are composed of laminar stretches consisting of $4: 2$ cycles in which the degree of alternation increases progressively until an intermittent sequence of one or more consecutive $1: 1$ cycles appears, after which there is a return to a stretch of $4: 2$ cycles. The average duration of the laminar phase consisting of successive 4:2 cyles decreases as $t_{s}$ decreases within the range of $t_{s}$ over which an irregular rhythm is produced. The fraction of action potentials getting through to the distal end of the cable is slightly greater than $1 / 2$ throughout this irregular zone, whereas it is exactly $1 / 2$ for the $4: 2$ rhythm. There is thus a paradoxical reduction in the degree of block produced by decreasing $t_{s}$ through $150 \mathrm{msec}$.

For $139 \mathrm{msec} \geq t_{s} \geq 105 \mathrm{msec}$, the irregular rhythms are replaced by a $3: 1$ rhythm (not shown). As $t_{s}$ is reduced in the range $105 \mathrm{msec}>t_{s} \geq 44 \mathrm{msec}$ in $1 \mathrm{msec}$ steps, one repeatedly sees transitions analogous to the $\{2: 1 \rightarrow 4: 2 \rightarrow$ irregular $\rightarrow 3: 1\}$ sequence described above: i.e. $\{n: 1 \rightarrow 2 n: 2 \rightarrow$ irregular $\rightarrow n+1: 1\}$, with $3 \leq n \leq 6$. For $43 \mathrm{msec} \geq t_{s} \geq 25 \mathrm{msec}$, one sees only irregular rhythms (except at $t_{s}=38 \mathrm{msec}$, where 


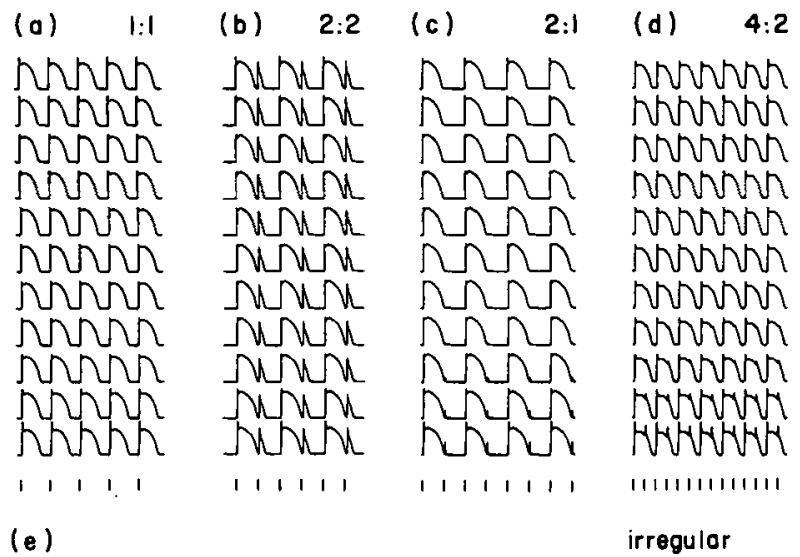

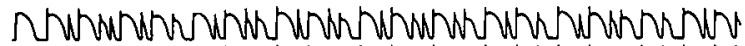

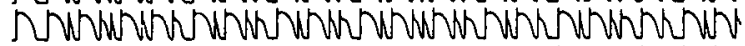

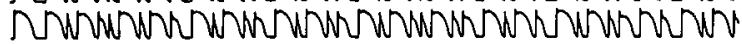

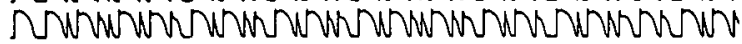

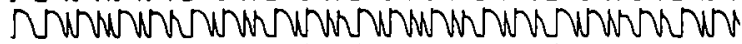

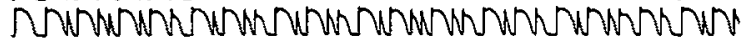

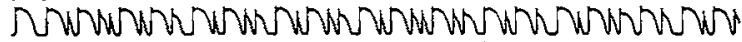

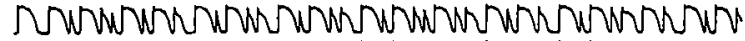

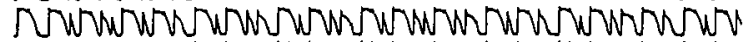
sowhow

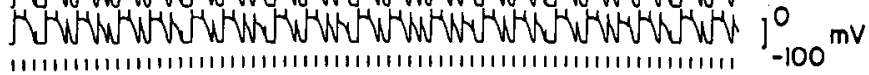

\section{I sec}

FIG. 1. Response of one-dimensional cable to periodic stimulation. (a), $1: 1$ rhythm, $t_{s}=400 \mathrm{msec}$, (b), 2:2 rhythm, $t_{s}=301 \mathrm{msec}$, (c), $2: 1$ rhythm, $t_{s}=290 \mathrm{msec},(\mathrm{d}), 4: 2$ rhythm, $t_{s}=151 \mathrm{msec}$, (e), irregular rhythm, $t_{s}=143 \mathrm{msec}$. In (b), the $A P D$ in the segment 90 alternates between 62 and $249 \mathrm{msec}$, and the conduction velocity in the middle of the strand between 62 and $67 \mathrm{~cm} \mathrm{sec}^{-1}$. (a)-(e), Bottom to top: stimulus pulses and transmembrane potential $(V)$ vs. time $(t)$ in segments $1,10,20, \ldots, 90,100$ of strand. Voltage traces are displaced vertically with an offset of $125 \mathrm{mV}$. Traces in (a)-(d) taken from near end of a 10-sec simulation run, at which point the transient due to initial conditions has largely damped out. Note the small-amplitude voltage responses in (c)-(e) that decrement in amplitude with distance down the strand. Calibration bar in (e) also refers to (a)-(d).

an 8:1 rhythm is seen). Finally, for $24 \mathrm{msec} \geq t_{s} \geq 11 \mathrm{msec}$, a $1: 0$ rhythm is encountered in which all stimuli are blocked, producing only local, spatially decrementing, subthreshold responses in the proximal part of the strand.

All the above simulations were started from the same initial conditions in all segments of the cable, corresponding to pacing the space-clamped membrane at $t_{s}=500 \mathrm{msec}$ (see Methods). When the initial conditions are changed to those corresponding to an infinite rest (i.e. all activation and inactivation variables set to their asymptotic values), the $\{2: 2 \rightarrow 2: 1\}$ transition occurs between $t_{s}=296$ and $297 \mathrm{msec}$ instead of between $t_{s}=293$ and $294 \mathrm{msec}$. Figure 2 shows an example at $t_{s}=295 \mathrm{msec}$, in which a 2:2 rhythm is seen starting from our standard initial 
(o)

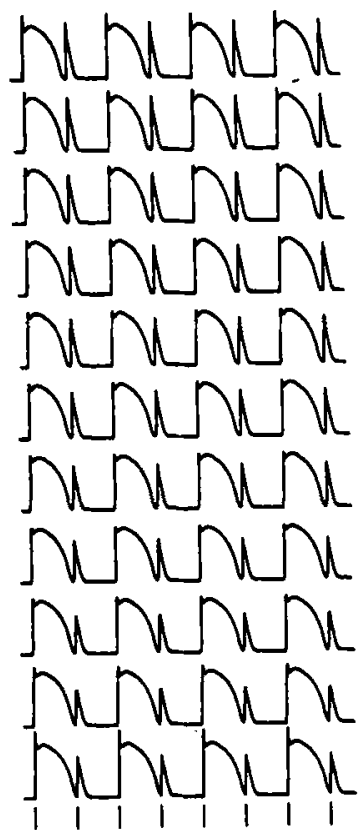

$2: 2 \quad(b)$

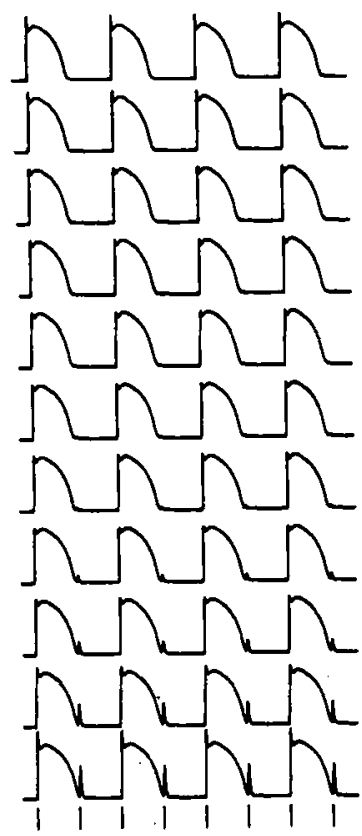

FiG. 2. Bistability in the response of the cable to periodic stimulation. $t_{s}=295 \mathrm{msec}$. (a) $, 2: 2$ rhythm, obtained starting numerical integration from standard initial conditions (given in Methods). (b), $2: 1$ rhythm, obtained starting numerical integration from asymptotic rest initial conditions. Traces taken from near end of a 10-sec simulation run in order to allow transients to dissipate. APD of first action potential at start of simulation run from which (b) is taken was 22 msec greater than than in (a). APD in segment 90 in the steady-state: 250 and $43 \mathrm{msec}$ (a), $254 \mathrm{msec}$ (b).

conditions [Fig. 2(a)], while a 2:1 rhythm occurs starting from asymptotic initial conditions [Fig. 2(b)]. Thus bistability-the simultaneous coexistence of two different periodic rhythms (viz 2:2 and 2:1) —is present between $t_{s}=293$ and $297 \mathrm{msec}$.

\section{Reduction to a One-dimensional Map}

There are several experimental reports showing that the response of a variety of cardiac preparations to periodic stimulation with a train of pulse stimuli can be accounted for by their response to premature stimulation with a single such stimulus (Moe et al., 1977; Scott, 1979; Guevara et al., 1981; Shrier et al., 1987; Chialvo et al., 1990; Guevara, 1990). In fact, it is clear that the various responses in Fig. 1 are due to the existence of refractoriness in the membrane. We therefore investigated the recovery properties of the cable using a premature stimulation protocol, which is a standard technique used in experimental cardiac electrophysiology. The system is paced at a basic cycle length long enough to produce a $1: 1$ rhythm and a premature stimulus applied every so often so as to probe the recovery or restitution process 
of the system, as characterized by some parameter describing the system (e.g. conduction velocity, action potential duration, upstroke velocity, etc....).

Figure 3(a) shows an example in which a premature stimulus delivered at a coupling interval $\left(t_{c}\right)$ of $400 \mathrm{msec}$ following the last action potential of the basic drive train produces an action potential with a significantly reduced action potential duration $(A P D)$. Initial condition corresponding to pacing at a basic cycle length of $500 \mathrm{msec}$ are used (see Methods). Figure $3(b)$ gives the $A P D$ restitution curve (Boyett \& Jewell, 1978 and references therein), which plots $A P D$ as a function of the recovery time $\left(t_{r}\right)$, both of which are measured at the distal end of the cable

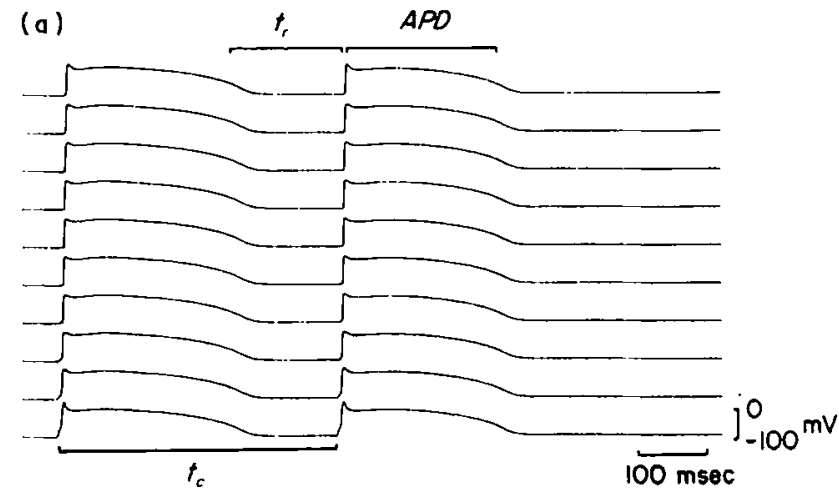

(b)

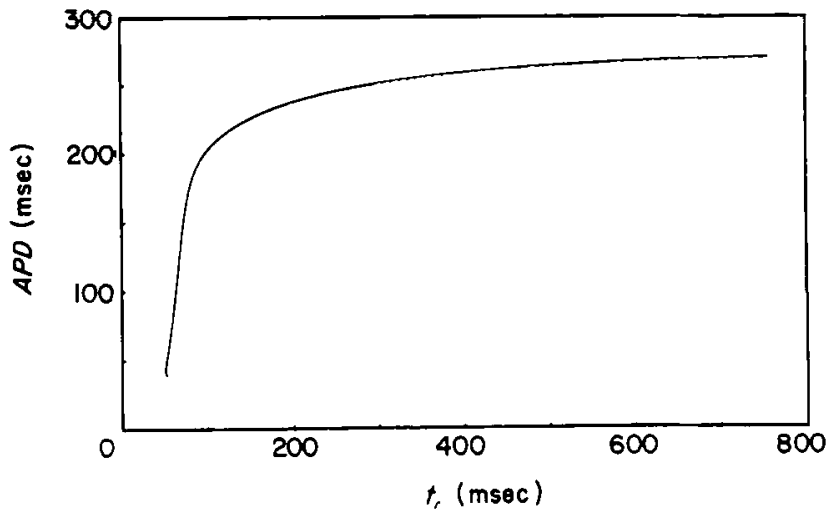

FiG. 3. (a), Response of one-dimensional cable to premature stimulation. The first action potential shown is the last one of the basic drive train. The second action potential is produced by injecting a premature stimulus into segment 1 of the strand at $t_{s}=400 \mathrm{msec}$. The coupling interval $\left(t_{\mathrm{s}}\right)$ is defined as the time from the onset of the last stimulus pulse of the basic drive train to the onset of the premature stimulus. The action potential duration $(A P D)$ is defined as the time from $-60 \mathrm{mV}$ on the upstroke to $-60 \mathrm{mV}$ on the repolarizing limb of the prematurely elicted action potential in segment 90 of the cable. The recovery time $\left(t_{r}\right)$ is defined as the time from $-60 \mathrm{mV}$ on the repolarizing limb of the last action potential of the basic train in segment 90 to $-60 \mathrm{mV}$ on the upstroke of the prematurely elicited action potential. Bottom to top: segments $1,10,20, \ldots, 80,90$. (b), Action potential duration restitution curve. $A P D$ is plotted as a function of $t_{r}$ for $t_{\mathrm{c}}$ changing in steps of $1 \mathrm{msec}$ in the range $292 \mathrm{msec}<t_{\mathrm{c}}<367 \mathrm{msec}$, $2 \mathrm{msec}$ steps for $367 \mathrm{msec}<t_{c}<545 \mathrm{msec}$, and $10 \mathrm{msec}$ steps for $545 \mathrm{msec}<t_{c}<1005 \mathrm{msec}$. 
(segment 90). The restitution curve shows that there is a monotonic decrease in $A P D$ with decreasing $t_{r}$ until $t_{r_{\text {min }}}(53.50 \mathrm{msec})$, the minimum recovery interval for which conduction is successful. This curve is fit very well $\left(r^{2}=0.996\right)$ by the sum of two exponentials, which agrees with the experimental finding in ventricular muscle (Elharrar \& Surawicz, 1983; Robinson et al., 1987). One obtains

$$
A P D=g\left(t_{r}\right)=A-B_{1} \exp \left[-t_{r} / \tau_{1}\right]-B_{2} \exp \left[-t_{r} / \tau_{2}\right], \quad t_{r}>t_{r_{\text {min }}},
$$

where $A=270 \mathrm{msec}, B_{1}=2441 \mathrm{msec}, B_{2}=90.02 \mathrm{msec}, \tau_{1}=19.60 \mathrm{msec}$, and $\tau_{2}=$ $200.5 \mathrm{msec}$. The asymptotic value of $A P D(\mathrm{~A})$ was obtained directly by setting initial conditions in the model to their asymptotic quiescent values and stimulating once to produce a single propagated action potential; it is not a result of the fitting procedure. On the scale of Fig. 3(b), the fit given by eqn (2) superimposes with the data shown there.

Making two assumptions, we now use the response to premature stimulation [Fig. 3 (b)] to predict the response to periodic stimulation (Figs 1 and 2). The first assumption is that during periodic stimulation at arbitrary $t_{s}$, the $A P D$ of any given action potential is controlled only by its immediately preceding recovery time and that this dependence is given by the $A P D$ restitution curve of Fig. 3(b). One can then write [Fig. 4(a)]

$$
A P D_{i+1}=g\left(t_{r_{i+1}}\right) \text {, }
$$

where $A P D_{i+1}$ is the $A P D$ of the $(i+1)$ st action potential, $t_{r_{i+1}}$ is its associated recovery time, and $g$ is the double-exponential function of eqn (2) describing the restitution curve of Fig. 3(b). From Fig. 4(a), it can be seen that $t_{s} \simeq A P D_{i}+t_{r_{i+1}}$, and so

$$
t_{r_{i+1}} \approx t_{s}-A P D_{i}
$$

Substituting eqn (4) into eqn (3), one has

$$
A P D_{i+1} \approx g\left(t_{s}-A P D_{i}\right) \text {. }
$$

We now make our second assumption: should one or more stimuli be blocked, producing only a spatially decrementing subthreshold response in the cable, the relationship between the $A P D$ of the subsequent action potential and its recovery time is not affected by the presence of the subthreshold response(s). One then has

$$
A P D_{i+1} \simeq g\left(n t_{s}-A P D_{i}\right)=f\left(A P D_{i}\right)
$$

if $(n-1)$ blocked stimuli occur in the recovery interval $t_{r_{i+1}}$ (i.e. $n$ is the smallest integer such that $n t_{s}-A P D_{i+1} \geqslant t_{r_{\min }}$ ).

Equation (6) was first derived by Guevara et al. (1984), but with $g$ extracted from the response during periodic stimulation runs carried out over a range of stimulation frequencies, and not from a premature stimulation protocol as described above. Nolasco \& Dahlen (1968) used a graphical iteration technique, extracting $g$ from the transient following a step change in rate. Thus, in both of these studies, the response to periodic stimulation was used to retrospectively account for that very response. Mahler \& Rogel (1970), who studied the contractile response of the heart, derived eqn (6) for the special case $n=1$, employing a premature stimulation protocol. 
(a)
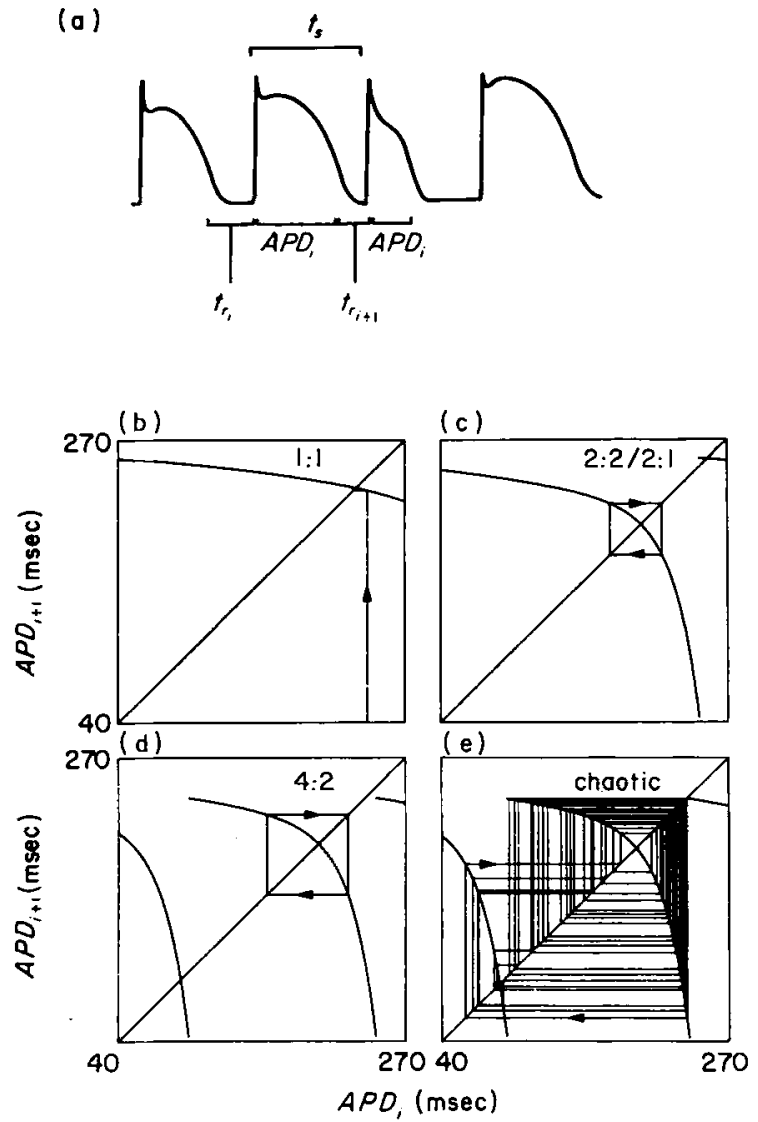

Fig. 4. (a) Schematic diagram used in deriving eqn (5), as described in text. Panels (b)-(e) show plots of the map of eqn (6) at four different values of $t_{s}$. Iterations are also shown, starting from an initial condition of $A P D_{i}=240 \mathrm{msec}$, which is close to the $A P D$ of the first action potential in the simulations of Fig. 1. The arrows give the direction of iteration. Transients are suppressed in (c)-(e). (b), $t_{s}=\mathbf{4 0 0} \mathrm{msec}$. Iterates asymptotically converge to the stable period-1 orbit, corresponding to a $1: 1$ rhythm. (c), $t_{s}=301 \mathrm{msec}$. Iterates converge to the stable period-2 orbit shown, corresponding to a 2:2 rhythm. For some initial conditions, iterates converge to the stable period-1 orbit on the right-hand branch of the map [ $n=2$ in eqn (6)], corresponding to a 2:1 rhythm. Bistability is thus present (see text). (d), $t_{s}=150 \mathrm{msec}$. A third branch has now appeared on the map corresponding to $n=3$ in eqn (6). The period-1 orbit on the right-hand branch $(n=2)$ in $(c)$ above has period-doubled, leading to a period-2 orbit on the middle branch here, corresponding to $4: 2$ rhythm. (e), $t_{s}=146 \mathrm{msec}$. Aperiodic orbit, visiting the leftmost $(n=1)$ and middle $(n=2)$ branches of the map. The rightmost $(n=3)$ branch has not yet intersected the line of identity $A P D_{i+1}=A P D_{i}$.

Equation (6) is a one-dimensional finite-difference equation or map, in that given $t_{s}$ and the function $g, A P D_{i+1}$ is a function $(f)$ of $A P D_{i}$ alone. From any starting value or initial condition $A P D_{1}$, one can calculate $A P D_{2}$; once $A P D_{2}$ is found, $A P D_{3}$ can then be calculated from $A P D_{2}$; and so on. In this fashion, one can iterate the map to determine the dynamics. 
Figures 4(b)-(e) show plots of $A P D_{i+1}$ vs. $A P D_{i}$ (i.e. the function $f$ describing the map) and iterations of the function $f$ for various $t_{s}$. The intersection of $f$ and the diagonal line of identity $\left(A P D_{i+1}=A P D_{i}\right)$ gives the steady-state(s). The iterations shown in Fig. 4(b) $\left(t_{s}=400 \mathrm{msec}\right)$ demonstrate that the stable-steady state or period-1 orbit at $A P D_{i}=A P D_{i+1}=231 \mathrm{msec}$ is asymptotically approached in an alternating manner. This steady-state corresponds to a $1: 1$ rhythm with an $A P D$ of $231 \mathrm{msec}$. As $t_{s}$ is decreased, the map moves down and to the left, so that the steady-state value of $A P D$ decreases and the alternating transient becomes more marked, as found in the cable simulations. Also, the slope of the map at the stable steady-state becomes more negative. Eventually, as this slope becomes more negative than -1 , the period-1 orbit becomes unstable and is replaced by a stable period- 2 orbit, corresponding to a 2:2 rhythm [Fig. 4(c)]. This period-2 orbit arises out of a period-doubling bifurcation (May, 1976; Thompson \& Stewart, 1986) that occurs at $t_{s}=301.8 \mathrm{msec}$.

The value of $t_{s}$ has by now become sufficiently small so that blocked stimuli can occur should $A P D_{i}$ be sufficiently large, thus making $t_{r_{i+1}}<t_{r_{\min }}$. The map thus has two branches: the left-hand branch obtained from eqn (6) with $n=1$, and the right-hand branch with $n=2$, corresponding to a single blocked stimulus preceding the $(i+1)$ st action potential. In Fig. $4(\mathrm{c})\left(t_{s}=301 \mathrm{msec}\right)$, for an initial condition $A P D_{1}>248 \mathrm{msec}$, the iterates converge to a period-1 orbit on the right-hand branch of the map at $A P D_{i+1}=A P D_{i}=254 \mathrm{msec}$, corresponding to a $2: 1$ rhythm. For $A P D_{1}<248 \mathrm{msec}$, the period-2 orbit illustrated, corresponding to a $2: 2$ rhythm, is approached asymptotically. Bistability is thus present on the map: iterates converge to one orbit or the other depending upon the initial condition (i.e $A P D_{1}$ ) chosen. The set of initial conditions ("basin of attraction") leading to each of the two orbits in Fig. $4(\mathrm{c})$ is a single interval $\left[g\left(t_{r_{\text {min }}}\right) \leq A P D_{1}<248 \mathrm{msec}\right.$ for the period-2 orbit; $270>A P D_{1}>248 \mathrm{msec}$ for the period-1 orbit]. In contrast, as $t_{s}$ is reduced, the basin of attraction of the period-2 orbit is split into two intervals; if $A P D_{1}$ is sufficiently large, the period- 2 orbit is asymptotically approached. As $t_{s}$ is decreased from the value used to produce the period-1 orbit on the $n=1$ branch of Fig. 4(b), the $n=2$ branch intersects the $A P D_{i+1}=A P D_{i}$ line of identity before the period-1 orbit on the $n=1$ branch period doubles. Thus, a $\{1: 1 \leftrightarrow 2: 1\}$ bistability is first seen which turns into the $\{2: 2 \leftrightarrow 2: 1\}$ bistability as $t_{s}$ is decreased further [Fig. 4(c)].

As $t_{s}$ is decreased still further, the following changes occur: the period- 2 orbit of Fig. 4(c) grows in amplitude (corresponding to an increased degree of alternation in $A P D)$, until at $t_{s}=297.94 \mathrm{msec}$, it collides with an unstable period-2 orbit and disappears in an abrupt fashion via a reverse saddle-node bifurcation (Thompson \& Stewart, 1986). However, the period-1 orbit on the $n=2$ branch (corresponding to the $2: 1$ rhythm) remains, and all initial conditions lead to this stable orbit. As $t_{s}$ is reduced further, the right-hand branch of Fig. $4(\mathrm{c})$ steepens, and once again there is a period-doubling bifurcation at $t_{s}=150.8 \mathrm{msec}$, in which the period-1 orbit on the $n=2$ branch is replaced by a period-2 orbit on that same branch [Fig. 4(d)]: the $2: 1$ rhythm is thus replaced by a $4: 2$ rhythm. By this point, the $n=3$ branch has already appeared, and can be seen at the extreme right-hand side of Fig. 4(d). The period- 2 orbit on the $n=2$ branch in Fig. 4(d) disappears at $t_{s}=148.9 \mathrm{msec}$ in 
a discontinuous way, unlike that on the $n=1$ branch [Fig. 4(c)], which disappeared via a saddle-node bifurcation. That is, as $t_{s}$ is decreased, the period-2 orbit on the $n=2$ branch [Fig. 4(d)] simply grows until, at $t_{s}=148.9 \mathrm{msec}$, it becomes too large to be supported by that branch and "falls off" the top left-hand edge of the branch. At this $t_{s}$, the $n=3$ branch does not yet intersect the line of identity, which would result in a $3: 1$ rhythm. There is thus no bistability between the $4: 2$ and $3: 1$ rhythms.
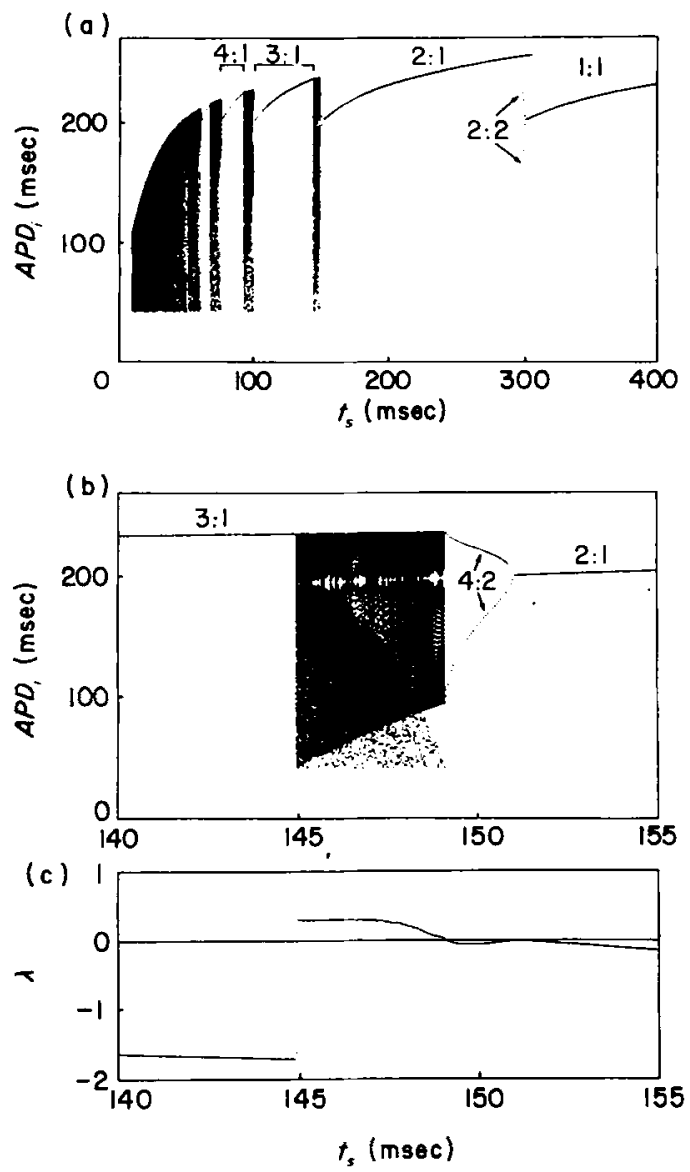

FiG. 5. (a), Bifurcation diagram ( $A P D_{1}$ vs. $t_{s}$ ) for $t_{s}=10-400 \mathrm{msec}$. At each $t_{s}$, eqn (6) was iterated 20000 times and the first 19800 iterates discarded to suppress transients due to initial conditions. Increment in $t_{s}$ was 1 msec. To demonstrate bistability, two initial conditions were used at each $t_{s}$ $\left(A P D_{1}=240\right.$ and $\left.270 \mathrm{msec}\right)$. (b), Expanded view of bifurcation diagram of (a) in range $t_{s}=140-155 \mathrm{msec}$. The period-doubling of the period-1 orbit on the $n=2$ branch (corresponding to the $2: 1$ rhythm of Fig. $1(c)$ ] to a period-2 orbit [corresponding to the $4: 2$ rhythm of Fig. 1(d)] is clearly shown, as is the transition from the $4: 2$ rhythm to aperiodic dynamics [Figs 1 (e) and 4(e)]. Increment in $t_{x}$ was $0.05 \mathrm{msec}$. (c), Liapunov number $(\lambda)$ calculated over the same range of $t_{s}$ as displayed in (b). $\lambda$ was calculated from eqn (7) with $N=20500$. The first 500 iterates were discarded to minimize the contribution to $\lambda$ of chaotic transients seen close to the 3:1 boundary (Grebogi et al., 1984). Computations shown in Figs 4 and 5 were carried out on an HP1000 minicomputer in double precision (approximately 15 significant decimal digits). 
In fact, at values of $t_{s}$ lying in the gap between values for which the $4: 2$ and $3: 1$ rhythms are seen, one obtains chaotic orbits: trajectories spiral outward on the $n=2$ branch in an alternating fashion and are injected onto the $n=1$ branch, only to be reinjected onto the $n=2$ branch after one or more iterations on the $n=1$ branch [Fig. 4(e)]. This behaviour is reminiscent of type-III intermittency as described by Pomeau \& Manneville (1980). Finally, at $t_{s}=144.9 \mathrm{msec}$, a stable period-1 orbit appears on the rightmost $(n=3)$ branch in Fig. 4(e) (i.e. 3:1 rhythm), with the concurrent abrupt disappearance of aperiodic dynamics. However, long chaotic transients resembling the nonperiodic orbit shown in Fig. 4(e) are present before the $n=3$ branch is approached, provided that the initial condition $A P D_{1}$ is chosen appropriately. The average lifetime of these "chaotic transients" decreases as one moves deeper into the $3: 1$ zone (Grebogi et al., 1984).

Further decrease in $t_{s}$ leads to the successive appearance of more branches from the right-hand edge of the range of the map, producing stable period- 1 orbits (i.e. $n: 1$ rhythm, $n \leq 6)$. These orbits period-double, producing $2 n: 2$ rhythms, and then go on to aperiodic orbits. For $t_{s}<50.33 \mathrm{msec}, n: 1$ or $2 n: 2(n \geq 7)$ orbits are not seen when $t_{s}$ is decreased in steps of $0.1 \mathrm{msec}$ : only aperiodic orbits are evident. Unlike the case in Fig. 4(e), some of these aperiodic orbits visit more than two branches of the map.

Figure 5(a) shows a bifurcation diagram $\left(A P D_{i}\right.$ vs. $\left.t_{s}\right)$ which summarizes the changes in steady-state dynamics of the map. Note the $\{1: 1 \leftrightarrow 2: 1\}$ bistability for $307.3 \mathrm{msec} \geq t_{s}>301.8 \mathrm{msec}$ and the $\{2: 2 \leftrightarrow 2: 1\}$ bistability for $301.8 \mathrm{msec} \geq t_{s}>$ $297.9 \mathrm{msec}$. Figure $5(\mathrm{~b})$ is an expanded view of the chaotic region between the $2: 1$ and $3: 1$ zones. The structures seen in this chaotic zones of the bifurcation diagram (the gradations in density) are a result of the shape of the map and the presence of an inherent discontinuity.

We have claimed above that the aperiodic behaviours shown in Figs 4(e) and $5(\mathrm{a})$ and (b) are manifestations of chaotic dynamics. We do so based upon calculation of the Lyapunov exponent $(\lambda)$

$$
\lambda=\lim _{N \rightarrow \infty} \sum_{i=1}^{N} \ln \left|\mathrm{d} f / \mathrm{d} A P D_{i}\right|_{A P D_{i}} \mid
$$

which measures the average exponential divergence of trajectories starting with very close initial conditions. A positive Lyapunov exponent indicates the existence of sensitive dependence on initial conditions, which is often taken as a definition of chaotic dynamics (Grebogi et al., 1983). Figure 5(c) gives $\lambda$ over the same range of $t_{s}$ as in Fig. 5(b), demonstrating that $\lambda$ is negative in ranges over which periodic orbits exist, but is positive in the irregular zone. In the other irregular zones of Fig. $5(a), \lambda$ is also positive (not shown). We have thus demonstrated that the irregular zones are manifestations of chaotic dynamics in the map.

\section{Comparison Between Cable Simulations and Map Iterations}

The above results indicate that the one-dimensional description derived from premature stimulation captures the dynamics of the partial differential equation 
very well, providing not only qualitative agreement (i.e. predicting bistability, perioddoubling bifurcations, chaos, etc), but good quantitative agreement with the simulations over a large range of $t_{s}$. Figure 6 provides a quantitative comparison, giving the range of $t_{s}$ over which a particular rhythm is seen in both the simulations and the iterations, demonstrating that the iterative technique predicts the existence of various rhythms to within a few msec of where they are actually found in the simulations. The only major discrepancy occurs at very small $t_{s}$, where a $1: 0$ rhythm is seen in the simulations, but not in the iterations. By continuity, a 1:0 rhythm must be seen for $t_{s}$ just larger than $10 \mathrm{msec}$ (at the current pulse amplitude used here), since injection of a constant bias current (i.e. $t_{s}=10 \mathrm{msec}$ ) of the same amplitude simply causes a depolarization of the membrane. The range of $t_{s}$ over which the $1: 0$ rhythm is seen decreases as the pulse amplitude used in the simulations is increased.

It is quite clear that eqn (6), being one-dimensional, can give only an approximation to the dynamics of the full-fledged partial differential equation describing the cable [eqn (1)], which is an 800-dimensional finite-difference equation in the discretized approximation used in the numerical integration routine. One can determine how well the system can be represented by a one-dimensional description by plotting a first-return map during irregular dynamics (Glass et al., 1984). Figure 7 shows an example, in which the square symbols are next- $A P D$ data points extracted from a numerical simulation corresponding to a $60-\mathrm{sec}$ long periodic stimulation run at $t_{s}=143 \mathrm{msec}\left[\right.$ Fig. 1(e)]: $A P D_{i+1}(1 \leq i \leq 230)$ in segment 90 is plotted as a function of the immediately preceding $A P D\left(A P D_{i}\right)$. The data points lie within two relatively thin bands, thus suggesting that the dynamics can indeed be approximated by a one-dimensional description. However, there is a slight degree of overlap between the bands, again indicating the higher-dimensional nature of the dynamics.

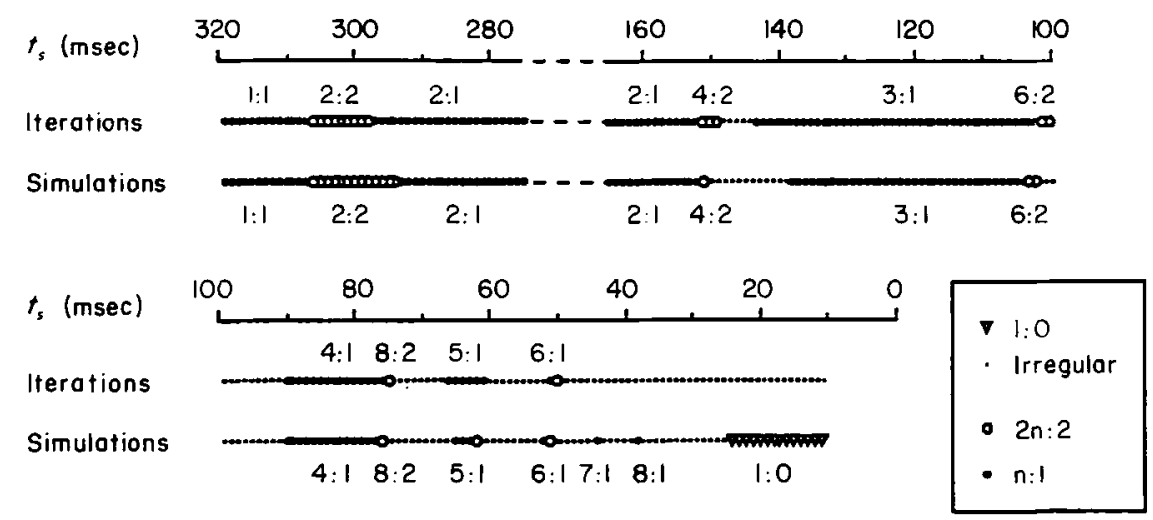

F1G. 6. Comparison between results of cable simulations and map iterations. Since 10 sec of activity in the cable was simulated, the number of iterations carried out at a given $t_{s}$ was the smallest integer just larger than $10000 / N t_{s}$, where $N$ is the average ratio of conducted beats to stimuli in a given simulation run. For $t_{s}<38 \mathrm{msec}, N=8$. $A P D_{1}=240 \mathrm{msec}$, which is close to the $A P D$ of the first action potential in the simulations. $t_{s}$ changed in 1 msec steps. $(\nabla), 1: 0 ;(\bullet)$, irregular; $(O), 2 n: 2 ;(O), n: 1$. 


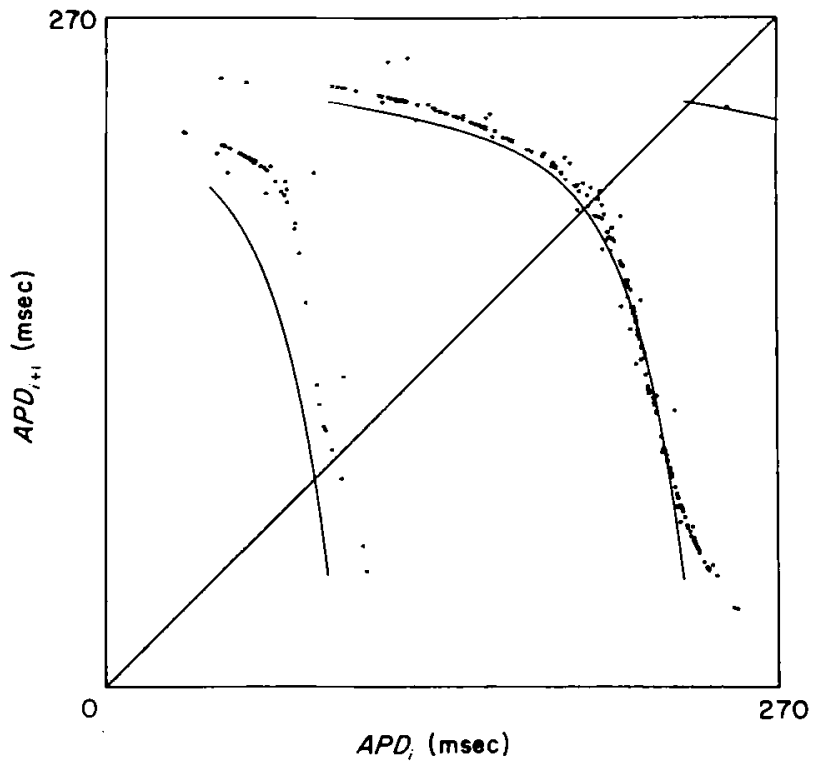

FIG. 7. The return map. Symbols give return map $\left(A P D_{i+1}\right.$ vs. $\left.A P D_{i}\right)$ obtained from numerical simulation of the cable equation at $t_{s}=143 \mathrm{msec}$ [see Fig. 1(e)] for $60 \mathrm{sec}$. Superimposed is the three-branched map [see Fig. 4(e)], eqn (6), derived from the response to premature stimulation, at the same $t_{s}$.

Also shown in Fig. 7 is the three-branched map derived from eqn (6) for $t_{s}=$ $143 \mathrm{msec}$, which predicts the existence of a $3: 1$ rhythm. Note that the points obtained from the cable simulations lie quite close to the map of eqn (6), especially along the middle $(n=2)$ branch. There are four main areas where the data points deviate from the map in Fig. 7.

(i) The data points falling along the $n=1$ branch of the map lie substantially above that branch. Since the points lie above the branch, this means that $A P D_{i+1}$, for a given $A P D_{i}$, is considerably larger than one would predict from the map. The reason for this deviation, as we shall now explain, is essentially that $A P D_{i}$ is very small $(<100 \mathrm{msec})$. The restitution curve from which the map shown in Fig. 7 was derived was established at a basic cycle length of $500 \mathrm{msec}$ (Fig. 3), when the $A P D$ of the action potential associated with the basic drive train was quite long. When the restitution curve is determined at a shorter basic cycle length, so that the $A P D$ of the action potential associated with the basic drive train is smaller, one finds that the $A P D$ of a premature beat elicited at a given $t_{r}$ is longer (see also Boyett \& Jewell, 1978; Elharrar \& Surawicz, 1983). When the basic cycle length is in the $1: 1$ zone (above $306 \mathrm{msec}$ ), this effect is small, since the $A P D$ s associated with the basic drive train in the $1: 1$ zone are all quite large. However, when the restitution curve is derived at a basic cycle length in the $2: 2$ zone and the premature stimulus follows the action potential with short duration, substantial effects may be seen. This is the reason why the data points lie above the $n=1$ branch in Fig. 7: $A P D_{i+1}$ is underestimated by the map, because $A P D_{i}$ is short. Thus, $A P D_{i+1}$ not only depends on $t_{r_{i}}$ 
(and therefore, during periodic stimulation, on $A P D_{i}$ implicitly), but on $A P D_{i}$ explicitly (see also Kaplan, 1989).

(ii) Data points also lie systematically above the left-hand end of the $n=2$ branch. The deviation away from the map is less than that for the $n=1 \mathrm{branch}$, and decreases as $A P D_{i}$ increases, being close to zero at $A P D_{i} \simeq 210-230 \mathrm{msec}$, which is very close to the $A P D$ of the action potential at the basic cycle length at which the restitution curve was determined. Thus, the reason for this deviation is exactly that detailed above for the $n=1$ branch: $A P D_{i}$ is shorter than the $A P D$ of action potentials in the basic drive train used for determining the restitution curve (and hence the map).

(iii) While most data points lie within two thin bands, there are a few exceptions. For example, two pairs of points lie at a considerable distance above the $n=1$ and $n=2$ bands. Upon inspection of the precursors of these points, one finds that both $A P D_{i}$ and $A P D_{i-1}$ were quite short. These points lie above the thin bands for a reason similar to that explained above for the thin bands lying above the map. The dependence on prior history extends past the immediately preceding action potential: $A P D_{i+1}$ seems not only to depend strongly on $A P D_{i}$, but on $A P D_{i-1}$ as well. This dependence is especially evident when $A P D_{i}$ and $A P D_{i-1}$ are both short.

(iv) The data points associated with the extreme right-hand-end of the $n=2$ branch lie increasingly above and to the right of that branch as $A P D_{i}$ increases. (There is a hint of similar behaviour on the $n=1$ branch). This is due to the fact that $A P D_{i+1}$ is very short for these points, indicating that $t_{r_{i+1}}$ is also extremely small. When $t_{r_{i+1}}$ is very close to the minimum recovery time, $t_{r_{\text {min }}}$, one begins to see a slowing of conduction velocity of the $(i+1)$ st action potential. The recovery time in the 90th segment, approximated by $t_{r_{i+1}} \simeq t_{s}-A P D_{i}$ [eqn (4) and Fig. $4(a)$ ], is then underestimated, since additional recovery time is provided by the extra conduction time. $A P D_{i+1}$ is thus longer than would be predicted from the map: data points therefore lie above the map. The additional recovery time provided by the slowing of conduction when recovery time is extremely short also should allow action potentials to conduct that would otherwise block. Note that the thin band of data points lying near the bottom end of the $n=2$ branch also extends more to the right in Fig. 7 than does the $n=2$ branch itself. Since conduction velocity slows appreciably only over a very short range of recovery times close to $t_{r_{\text {min }}}$, the deviation from the map is seen only over a relatively short range of $A P D_{i}$.

\section{Discussion}

\subsection{COMPARISON WITH EXPERIMENTAL RESULTS}

As far as we are aware, no systematic laboratory experiments corresponding to the numerical cable simulations presented above have been carried out on ventricular muscle. However, there is experimental evidence for many of the phenomena seen.

(i) The transition $\{1: 1 \rightarrow 2: 2\}$, which is the result of a period-doubling bifurcation, has been observed in many studies on ventricular muscle preparations of various sorts, including papillary muscle (Hoffman \& Suckling, 1954; Boyett \& Jewell, 1978), quiescent and spontaneously beating aggregates of embryonic chick ventricular cells (Guevara, 1984; Guevara et al., 1984, 1990), and isolated rabbit ventricular cells 
(Guevara et al., 1989). In the latter two sets of studies, the transition $\{2: 2 \rightarrow 2: 1\}$ was also seen. Transisent alternation is often seen in studies on ventricular muscle paced at high rates (see Guevara, 1984 for references), and has been described in modelling work on Purkinje fibre (de Beer, 1977). Transient alternation is essentially due to the fact that the period-1 orbit in Fig. 4(b) lies on a branch with negative slope: thus, the approach to the fixed point has to be alternating. A similar situation is found in spontaneoulsy beating aggregates, where one obtains a non-invertible circle map having a region of negative slope (Guevara \& Shrier, 1990).

In the majority of the experimental studies mentioned above, the focus was on the alternation of action potential duration during the $2: 2$ rhythm. However, there is also a simultaneous beat-to-beat alternation in conduction velocity, for which there is experimental evidence in ventricular muscle (see Guevara, 1984 for references). Since one can obtain a beat-to-beat alternation in conduction time in a one-dimensional cable [Fig. 1(b)], it is perhaps not necessary to postulate the existence of longitudinal dissociation of the conduction system ("dual pathways") to obtain this effect in all cases where it is observed in experimental work.

(ii) The transition $\{2: 1 \rightarrow 4: 2\}$, which is the result of yet another period-doubling bifurcation, has been seen in spontaneously beating aggregates (Guevara, 1984; Guevara et al., 1990), in single guinea-pig ventricular cells at a raised external potassium concentration (Hescheler \& Speicher, 1989), and in rabbit ventricular cells at low temperature (Guevara et al., 1989). In the last mentioned case, the transition $\{3: 1 \rightarrow 6: 2\}$ was also described.

(iii) The presence of chaos following $1: 1,2: 2,2: 1$, and $4: 2$ rhythms has been documented in isolated guinea-pig ventricular cells at an elevated external potassium concentration (Hescheler \& Speicher, 1989), and in isolated rabbit ventricular cells at low temperature (Guevara \& Jeandupeux, unpublished). In addition, a very recent report on strands of Purkinje fibre reports the transition $\{4: 2 \rightarrow$ chaos $\}$ [Chialvo et al., 1990: Fig. 1(b)]. This chaotic rhythm however consists of mixtures of $4: 2$ and $3: 1$ cycles, and does not contain any $1: 1$ cycles. We have not seen such traces in our simulations, changing $t_{s}$ in 1 msec steps. The transition $\{3: 1 \rightarrow 6: 2 \rightarrow$ chaos $\}$ has been reported in the propagated action potential of the squid giant axon (Matsumoto et al., 1987a,b).

(iv) The 1:0 rhythm seen at very high stimulation rates is a consequence of post-repolarization refractoriness and has also been seen in shortened strands of Purkinje fibre (Chialvo \& Jalife, 1987).

(v) The $\{2: 2 \leftrightarrow 2: 1\}$ bistability (and consequent hysteresis) present in the simulations (Fig. 2) and the map [Fig. 4(c)] can be seen in isolated ventricular cells (Guevara et al., 1989). A different kind of $\{2: 2 \leftrightarrow 2: 1\}$ bistability was described a long time ago by Mines (1913), who studied a ring cut from the auricles of a tortoise. However, the phenomenon in that instance depends on the distributed, inhomogeneous nature of the preparation. The $\{1: 1 \leftrightarrow 2: 1\}$ bistability predicted by the map, but not seen in the simulations, was also observed in the pioneering report of Mines (1913), who studied the atropinized ventricle of the frog; many years later it was redescribed in spontaneously beating aggregates of ventricular cells (Guevara, 1984; Guevara et al., 1990) and quiescent isolated ventricular cells (Guevara et al., 
1989). Mines, who used extracellular recording techniques, did not describe a $2: 2$ rhythm in his experiments. In this regard, one should note that it is possible to obtain a direct transition from a $1: 1$ to a $2: 1$ rhythm should the slope of the left-hand branch of the map in Fig. 4(c) not become steep enough to generate a period-doubled orbit (Guevara et al., 1989; Kaplan, 1989). Iterations using eqn (6), but with the function $g$ of eqn (3) extracted from periodic stimulation runs, and not from a premature stimulation protocol, retrospectively predicted the existence of both the $\{1: 1 \leftrightarrow 2: 1\}$ and $\{2: 2 \leftrightarrow 2: 1\}$ bistabilities in quiescent aggregates (Guevara et al., 1984); however, no experimental search was carried out for these phenomena in that preparation.

The bistability between two different $2: 2$ rhythms described by Nolasco \& Dahlen (1968) - see also Mahler \& Rogel (1970) - is not seen on the map considered here. However, as described above, the stable period-2 orbit of Fig. 4 (c) generating the $2: 2$ rhythm is destroyed by collision with an unstable period-2 orbit as $t_{s}$ is decreased [Fig. 8(a)]. This unstable period-2 orbit arises in a discontinuous fashion upon decrease in $t_{s}$. However, should the minimum possible $A P D$ in Fig. 4(c) be decreased, so that left-hand branch of the map in Fig. 4(c) will be extended downwards, this unstable period- 2 orbit arises via a saddle-node bifurcation in which a stable period-2 twin orbit is also born [Fig. 8(b)]. Thus, a change in the parameters describing the restitution curve would be all that would be needed to obtain the coexistence of two $2: 2$ rhythms. While a premature beat or a pause in stimulation can convert one 2:2 rhythm into another $2: 2$ rhythm in the ischaemic ventricle (Hashimoto et al., 1984 and references therein), the degree to which this particular bistability might be due to spatial interactions is not clear at the present time. This existence of two stable periodic orbits on the same branch of the map is possible since its Schwarzian derivative can be positive. In such maps, it is also possible to have the coexistence of a $1: 1$ and a 2:2 rhythm or of a $1: 1$ rhythm and chaos (Mayer-Kress \& Haken, 1982). While we know of no experimental evidence for these two bistabilities in

(a)

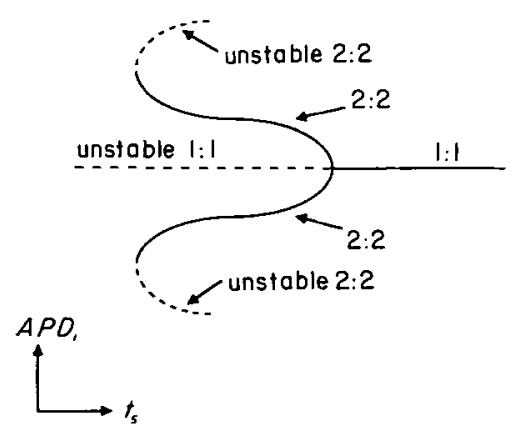

(b)

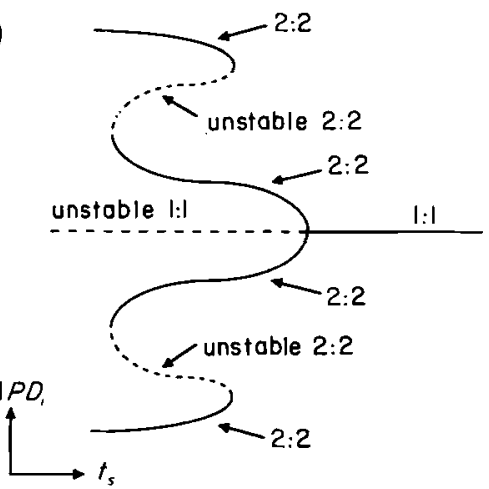

FIG. 8. Schematic bifurcation diagrams. (a), Bifurcation diagram illustrating how period-2 orbit of Fig. 4(c) (corresponding to a $2: 2$ rhythm) is destroyed. (b), Alternative bifurcation diagram showing how it is possible to obtain coexistence of two stable period-2 orbits (corresponding to two different $2: 2$ rhythms). Solid lines indicate stable orbits, dashed lines unstable orbits. Saddle-node bifurcations occur at turning points in (a) and (b). See text for further description. 
space-clamped systems, there is evidence for the coexistence of $1: 1$ and $2: 2$ rhythms in distributed systems (Mahler \& Rogel, 1979) and it is well known that normal sinus rhythm and ventricular fibrillation can coexist, with one being converted into the other with a fibrillating or defibrillating shock.

In the cable simulations, we did not find the $\{2: 1 \leftrightarrow 1: 1\}$ bistability predicted to exist from the iterations of the map. This could be because the bistability does not exist in the cable equation, or because we only investigated two sets of initial conditions, and the bistability is predicted to exist over a range of $t_{s}$ that is only a few msec wide. One practical point in simulation work is that it is very difficult, and expensive, to carry out a systematic search for bistability, since this involves, at each $t_{s}$, making many simulation runs from a large number of initial conditions. Thus we cannot rule out the possibility that there exist bistabilities in the cable equations other than those we have described above. In this respect, consideration of the map can be most useful in predicting the existence of the bistability and in narrowing down the range of initial conditions to be searched. In fact, it was the finding of the $\{2: 2 \leftrightarrow 2: 1\}$ and $\{2: 2 \leftrightarrow 1: 1\}$ bistabilities on the map that prompted our search for them in the simulations. However, as in the case of the simulations, the search for bistability on the maps is also time-consuming, and we cannot guarantee that bistabilities other than those we have described are not supported by the map, e.g. in Fig. 7, there is bistability between the period-1 orbit corresponding to a 3:1 rhythm and a chaotic rhythm. The existence of transient chaos also creates computational limitations: an orbit that appears chaotic in a finite-time computation might actually be transient chaos (Grebogi et al., 1984).

In many of the experimental findings listed above, an effectively isopotential preparation is used-isolated single ventricular cells or aggregates of small embryonic cells. Thus, a systematic experimental study on the propagated ventricular action potential is needed to determine the extent to which the rhythms predicted to exist in the cable simulations do in fact occur.

\subsection{REDUCTION TO A ONE-DIMENSIONAL DESCRIPTION}

While period-doubling and chaotic behaviour have previously been demonstrated in numerical simulations of partial differential equations (e.g. Moore et al., 1983), this is the first example that we know of in which a direct reduction to a onedimensional description has been successful in capturing the dynamics, since previous reductions have involved the intermediate step of reduction to a system of ordinary differential equations (e.g. Moore et al., 1983; Kahlert \& Rössler, 1984). However, since in certain instances the asymptotic behaviour of partial differential equations can be reduced to consideration of a set of ordinary differential equations, their "inertial form" (Foias et al., 1988; Temam, 1988), and since it is well-known that ordinary differential equations often have dynamics that is well approximated by a one-dimensional description, it is perhaps not surprising that a one-dimensional description works so well in this instance. However, in one study on a delaydifferential equation, which like the cable equation is of infinite dimension, it was found that a one-dimensional map derived in the singular perturbation limit did 
not have the same bifurcation structure as the delay-differential equation itself (Mallet-Paret \& Nussbaum, 1986).

The one-dimensional description works here mainly because the existence of a blocked stimulus does not significantly affect the activity in the distal part of the cable, since the subthreshold response decays electronically rapidly with distance [Fig. 1(c), (d) and (e)]. This is not the case if one considers the non-propagated Beeler-Reuter action potential.

Although the one-dimensional description works very well in accounting for the various rhythms observed (Fig. 6), Fig. 7 shows that there is still room for improvement, since there are systematic deviations of the data points (obtained during periodic stimulation) from the map (obtained from premature stimulation). Our analysis of the discrepancies suggests two main ways in which the one-dimensional description applied above can be improved and extended in future work. First, Fig. 7 demonstrates that $A P D_{i+1}$ depends not only on $t_{r_{i+1}}$ (and thus indirectly on $A P D_{i}$-Fig. 4), but also directly on $A P D_{i}$. Thus $A P D_{i+1}$ will have to be made a function of both $t_{r_{i+1}}$ and $A P D_{i}$. This extension of the theory could be carried out by determining the restitution curve at several different basic cycle lengths as has been done in experimental work (Boyett \& Jewell, 1978; Elharrar \& Surawicz, 1983), and interpolating. Second, one will have to include the effect of conduction time on recovery time in the distal segments of the cable, since Fig. 7 shows that this is an appreciable effect at very short recovery times. This could be accomplished by determining a restitution curve for conduction velocity, and incorporating this into the finite-difference equation in a manner similar to that recently utilized in the analysis of experiments on Purkinje fibre (Chialvo et al., 1990). Incorporation of both of the above effects should produce much better agreement between simulations and iterations, but at the cost of losing the one-dimensional nature of the theory.

\subsection{ROUTE TO CHAOS}

The route to chaotic dynamics in the map as $t_{s}$ is decreased is not the commonly described one involving an infinite cascade of period-doubling bifurcations (May, 1976): only two such bifurcations occur (Figs 4 and 5). This previously undescribed route occurs because of the presence of a discontinuity in the map, which is in turn a direct consequence of the fact that there is a true discontinuity in the response to premature stimulation [Fig. 2(b)] arising from the all-or-none nature of propagation in a cable (Miller \& Rinzel, 1981). The only equilibrium point present in the phase space of the Beeler-Reuter system of ordinary differential equations is not a saddle point. Thus, in the space-clamped or non-propagated action potential model, one would not obtain such a discontinuous map, since the response of the membrane is then not all-or-none, due to the absence of any saddle points (Clay et al., 1984). In that instance, one would not expect a direct transition from a $4: 2$ rhythm to chaos; rather, a period-4 orbit corresponding to an $8: 4$ rhythm might then be expected to arise out of a period-doubling of the period- 2 orbit corresponding to a 4:2 rhythm. Indeed, there is some recent experimental evidence for the transition 
$\{4: 2 \rightarrow 8: 4\}$ in periodically stimulated ventricular cells (Hescheler \& Speicher, 1989), and period-8 rhythms are seen in a simple two-variable excitation-refractoriness model of periodically forced space-clamped excitable tissue (Feingold et al., 1988). We have not been able to find evidence of any such higher-order period-doubled orbits in the simulations of Fig. 1 . To check for the existence of these orbits would involve changing $t_{s}$ in very small fractions of a millisecond, since the period- 4 orbit is found at $t_{s}=151 \mathrm{msec}$, and not at $t_{s}=150$ or $152 \mathrm{msec}$. In that case, one would also probably have to improve the accuracy of the numerical integration scheme. In an earlier study involving sinusoidal current stimulation of the space-clamped Beeler-Reuter membrane, only period-1, 2 and 4 rhythms were described; a period- 8 rhythm was not seen before the onset of chaotic dynamics (Jensen et al., 1984). A similar finding occurs in the response to a pulsatile input (Vinet et al., 1990). One would not expect such higher-order period-doubled rhythms to exist in the cable simulations provided that propagation in a cable of finite length is indeed all-or-none during periodic stimulation, since in that case there would still be a true discontinuity present in the response (as the return map of Fig. 7 might suggest). However, we are not aware of any such theoretical result. Finally, one should also keep in mind that the full nature of the bifurcation sequence is often not revealed in a oneparameter bifurcation analysis, and that an incomplete period-doubling cascade can occur in one-dimensional maps with non-neative Schwarzian derivative (MayerKress \& Haken, 1982) and in maps of dimension higher than one (Arneodo et al., 1983).

All of the above assumes that ventricular tissue is accurately represented by models of the Hodgkin-Huxley type, which are deterministic and continuous. However, there are stochastic effects present in cardiac tissue, since the electrical activity is generated by a population of channels in the membrane, which are presently thought to open and close in a random fashion. In addition, there is recent experimental evidence for a true all-or-none threshold for excitation of a prematurely elicited action potential in well-polarized, effectively space-clamped ventricular aggregates (Guevara et al., 1986: fig. 4), in the absence of any saddle points in a Hodkin-Huxley-type model of the aggregate (Clay et al., 1984). Thus, a study on propagation in an inherently stochastic model is indicated.

The sequence of transitions $\{1: 1 \rightarrow 2: 2 \rightarrow 2: 1 \rightarrow 4: 2 \rightarrow$ chaos $\rightarrow 3: 1 \rightarrow 6: 2 \rightarrow$ chaos $\rightarrow \ldots\}$ described above in the propagated ventricular action potential might not be universal, since it appears that, in a piecewise linear approximation to the class of maps studied here, the first transition to chaos can occur after the 3:1 rhythm is seen (Kaplan, 1989). In addition, it is also possible to obtain the transitions $\{1: 1 \rightarrow$ Wenckebach $\rightarrow 3: 2 \rightarrow$ reverse Wenckebach $\rightarrow 2: 1 \rightarrow \ldots)$ and $\{1: 1 \rightarrow 2: 1\}$ in a model of the propagated action potential in Purkinje fibre (Guevara, 1988). While, as mentioned above, the two-branched maps studied here have negative slope everywhere and can produce the $\{1: 1 \rightarrow 2: 1\}$ transition (with bistability), the transition involving Wenckebach rhythms involves a 2-branched map in which both branches have positive slope everywhere (Shrier et al., 1987; Guevara, 1990).

Finally, for a homogeneous cable of semi-infinite length, only $1: M$ rhythms are possible in the absence of supernormal conduction (Miller \& Rinzel, 1981). Many 
of the non-1 : $M$ rhythms seen in the heart thus hinge upon the fact that the ventricular wall is only a few space constants thick.

\subsection{CONNECTIONS WITH TYPE III INTERMITTENCY}

The irregular behaviour in the simulations [Fig. 1(e)] and maps [Fig. 4(e)] strongly resembles type 111 intermittency (Pommeau \& Manneville, 1980; Mayer-Kress \& Haken, 1982; Dubois et al., 1983), in that there is a long laminar phase with a progressively increasing level of alternation followed by a relatively short irregular "burst" and then re-injection into a new laminar phase. The only biological example that we know of in which type III intermittency has been described is in the case of the propagated action potential in the squid giant axon, where one can see the transition $\{3: 1 \rightarrow$ type III intermittent chaos $\}$ as stimulus amplitude is increased at fixed $t_{s}$ (Matsumoto et al., 1987 b: fig. 6). In other experiments on squid axon, the route to chaos is similar to that we have described above, in that one sees $\{3: 1 \rightarrow 6: 2 \rightarrow$ chaos\} (Matsumoto et al., 1987b: fig. 3). In the bifurcation diagram of Fig. 9(a), which represents the type III intermittency results of Matsumoto et al. (1987b), an unstable $6: 2$ rhythm coexists with the stable $3: 1$ rhythm (there is experimental evidence for this coexistence, Matsumoto et al., 1987b: fig. 6(c)]. As the stimulus pulse amplitude is increased beyond the bifurcation point, one obtains a direct transition from a 3:1 rhythm to type III intermittent chaos. The definition of type III intermittency hinges upon there being a subcritical period-doubling bifurcation, such as that shown in Fig. 9(a), in which the onset of chaos occurs simultaneously with the period-doubling bifurcation (Pomeau \& Manneville, 1980). Since such a subcritical bifurcation does not occur in our simulations or maps, but rather a supercritical bifurcation [Fig. 9(b)], type III intermittency is not the mechanism for producing chaos above. However, our results [Fig. 9(b)] are in some sense close to type III intermittency [Fig. 9(a)], in that a change in the parameters describing the restitution curve would change our bifurcation diagram from that shown in Fig. 9(b) to that shown in Fig. 9(c), where there is a discontinuous appearance of an unstable period-2 orbit as $t_{s}$ is reduced, as in Fig. 8(a). In fact, Fig. 5(b) shows that the $\{2: 1 \rightarrow 4: 2 \rightarrow$ chaos $\}$ bifurcation diagram is close to this form, i.e. the turning-point associated with the saddle-node bifurcation in Fig. $9(\mathrm{c})$ is almost attained prior to the appearance of chaos. Should the range of $t_{s}$ over which the stable 6:2 rhythm seen in Fig. 9(c) be progressively reduced, the limiting case of Fig. 9(a), i.e. type III intermittency, would be approached.

\subsection{CONNECTIONS WITH CIRCLE MAPS}

Earlier, when we compared the results of our simulations with the resuits from experimental work, we made reference to experiments in which a spontaneously active preparation was studied. For example, the transitions $\{1: 1 \rightarrow 2: 2 \rightarrow 2: 1\}$ and $\{2: 1 \rightarrow 4: 2\}$ are seen in periodically driven spontaneously beating aggregates of ventricular cells (Guevara, 1984; Guevara \& Shrier, 1990; Guevara et al., 1990). In a fashion similar to that described above, the response to premature stimulation 
$(0)$

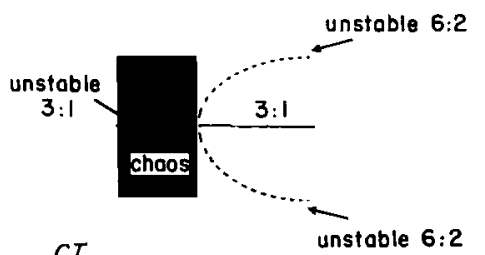

(b)

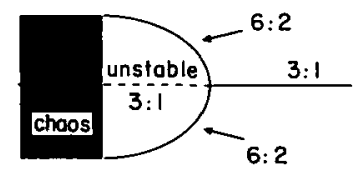

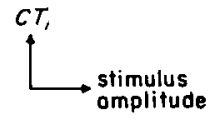
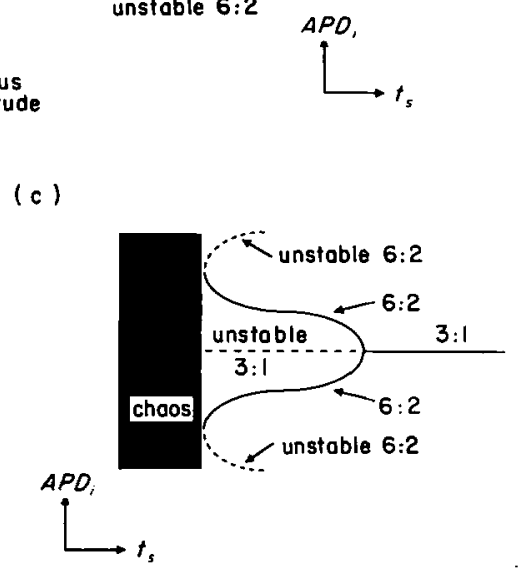

FIC. 9. Schematic bifurcation diagrams. (a), Bifurcation diagram illustrating type 111 intermittency of Matsumoto et al. $(1987 \mathrm{~b})$ in squid giant axon. $C T_{i}$ is the conduction time of the $i$ th action potential to propagate down the axon. (b), Bifurcation diagram illustrating transition from $3: 1$ rhythm to chaos in Figs 5(a) and (6), Bifurcation diagram obtained from that in (b) by modifying $A P D$ restitution curve. Solid lines indicate stable orbits, dashed lines unstable orbits. Saddle-node bifurcation occurs at turning point in (c). See text for further description.

("phase-resetting") can be investigated, and a map derived and iterated. Since the preparation is spontaneously oscillating, that map is a continuous circle map, rather than a multi-branched interval map as in this study. In such a circle map, which is of topological degree zero at a current pulse amplitude high enough to obtain the sequence of transitions $\{1: 1 \rightarrow 2: 2 \rightarrow 2: 1 \rightarrow 4: 2\}$, the $\{1: 1 \rightarrow 2: 2\}$ and $\{2: 1 \rightarrow 4: 2\}$ transitions are again the result of two consecutive period-doubling bifurcations (Guevara, 1984; Guevara \& Shrier, 1990). The transition $\{2: 2 \rightarrow 2: 1\}$ is due to a smooth shift in the position of a period-2 orbit (Guevara \& Shrier, 1990: fig. 6), whereas the transition in Fig. 4(c) is due to moving from a period-2 orbit on the $n=1$ branch to a period-1 orbit on the $n=2$ branch. Similar behaviours occur in a very simple limit-cycle model when type zero phase-resetting occurs (Guevara \& Glass, 1982). It is not surprising that results like those we have described above are found in a spontaneously active preparation, since, at the high stimulation rates needed to obtain the phenomena, the membrane potential spends little or no time in the phase of spontaneous diastolic depolarization. The saddle-node bifurcation producing two period-2 orbits [Fig. 8(b)] has also been described in two-extrema degree-0 maps (Coste \& Peyraud, 1982; Bélair \& Glass, 1985). Thus, while there 
are similarities in the bifurcation structure of degree- 0 circle maps and two-branched interval maps, a comprehensive comparative study is yet to be made.

\subsection{THE APD RESTITUTION CURVE}

The $A P D$ restitution curve of Fig. 3(b) is fit very well by a sum of two exponentials. While an early modelling study on Purkinje fibre (de Beer, 1977) and an early experimental study on ventricular muscle (Boyett \& Jewell, 1978) found that the $A P D$ restitution curve could be fit by a single exponential function, later experimental studies on ventricular muscle (Elharrar \& Surawicz, 1983; Guevara et al, 1984; Robinson et al., 1987), and Purkinje fibre (Elharrar \& Surawicz, 1983) used a double-exponential fit, finding two time constants in the ranges $43-66 \mathrm{msec}$ and 653-3000 msec for ventricular muscle at short basic cycle lengths ( $\leq 500 \mathrm{msec}$ ). In contrast, in our modelling study, we find time constants of 20 and $201 \mathrm{msec}$. The absence in our work of the longer time constant found in the experimental work $(653-3000 \mathrm{msec})$ indicates that it is probably due to some factor(s) not present in the Beeler-Reuter model. Indeed, it has been attributed to the $\mathrm{Na}^{+}-\mathrm{K}^{+}$pump and to ion accumulation, neither of which comes into play in the Beeler-Reuter model. It is thus not surprising that we do not find the longer time constant. Our results suggest that the single-exponential process described by the shorter time constant (43-66 msec) in the experimental work might actually be better represented by a sum of two exponentials.

The ionic mechanisms underlying restitution of action potential duration remain undetermined. Various authors have suggested roles for $I_{\mathrm{Na}}, I_{x 1}, I_{s}$, the transient outward current, the $\mathrm{Na}^{+}-\mathrm{K}^{+}$pump, and intracellular and extracellular ion accumulation and depletion. It is already clear that both $I_{s}$ and $I_{x 1}$ are involved in controlling the recovery process in the Beeler-Reuter model (Beeler \& Reuter, 1977; Drouhard \& Roberge, 1982). In particular, the long time constant for deactivation of $I_{x 1}$ causes significant amounts of $I_{x 1}$ to flow during an action potential elicited at a very short $t_{r}$, thus producing a dramatic shortening of $A P D$ (Drouhard \& Roberge, 1982: fig. 8). However, the time constant for the restitution of the duration of the action potential plateau at short $t_{r}$ agrees very well with that for reactivation of $I_{s}$ (Gettes \& Reuter, 1974). The experimental value of $30 \mathrm{msec}$ is of the same order of magnitude as our faster time constant. Thus further work, both experimental and modelling, needs to be carried out to elucidate the genesis of the $A P D$ restitution curve.

It is important to determine the factors governing the form of the $A P D$ restitution curve, since its exact shape is important in controlling the bifurcation structure of Fig. 5(a). Three examples were mentioned above in which a change in this shape changes the bifurcation structure, producing: (i) a direct $\{1: 1 \rightarrow 2: 1\}$ transition rather than the $\{1: 1 \rightarrow 2: 2 \rightarrow 2: 1\}$ transition; (ii) bistability between two $2: 2$ rhythms; and (iii) a direct $\{4: 2 \rightarrow 3: 1\}$ transition rather than the $\{4: 2 \rightarrow$ chaos $\rightarrow 3: 1\}$ transition. Finally, if the connection between alternans (and higher-order period-doubled rhythms?) and arrhythmias is a causal one, it might be possible in the furture to reduce the incidence of arrhythmias by pharmacologically modifying the steep 
left-hand portion of the restitution curve of Fig. 3(b), which controls the bifurcation sequence.

\subsection{IMPLICATIONS FOR VENTRICULAR ARRHYTHMIAS}

It has been known for many years that the $2: 2$ rhythm of alternans can be seen during myocardial ischaemia, hypothermia, and fast pacing of the ventricle, often immediately preceding the phase of induction of malignant ventricular arrhythmias such as tachycardia and fibrillation (Adam et al., 1982, 1984; Smith et al., 1988). There are also very recent reports of $4: 4$ rhythms in ventricular tissue during ischaemia (Dilly \& Lab, 1988), hypothermia (Kaplan, 1989), and fast pacing (Savino et al., 1989). These three reports of $4: 4$ rhythms are the only ones of which we are aware in situations predisposing to ventricular arrhythmias. The relative rarity of $4: 4$ with respect to $2: 2$ rhythms is probably connected with the fact that period-4 rhythms are seen over a much smaller range of the bifurcation parameter [Fig. 5(a)]. The simulations presented above show that the sequence $\{1: 1 \rightarrow 2: 2 \rightarrow 2: 1 \rightarrow 4: 2 \rightarrow$ chaos\} occurs in a model of the propagated ventricular action potential. We therefore suggest that there might be a causal link between a finite number of period-doubling bifurcations and the arrhythmias seen following ischaemia and other interventions. The number of such bifurcations might be as small as one or two; indeed, if the right-hand branch in Fig. 4(c) could come in more steeply, there would be a direct transition from a 2:2 rhythm to chaos. A direct transition from $2: 2$ rhythm to chaos as $t_{s}$ is decreased has been described recently in experimental work on Purkinje fibre (Chialvo et al., 1990: fig. 2), where it has been ascribed to a non-monotonicity of the $n=1$ branch of the map. In fact, should supernormal conduction be present, one can obtain a single-branched non-monotonic map in which there can be one or more period-doubling bifurcations, leading to $2: 2,4: 4,8: 8, \ldots$ rhythms (Guevara, 1990: fig. 12(c)]. In the class of such maps, it is possible to obtain chaotic dynamics following a single period-doubling bifurcation (Tresser et al., 1980).

To model ventricular arrhythmias caused by ischaemia, hypothermia, etc, some parameter(s) other than $t_{s}$ should be changed in the simulations, which should also be extended to three spatial dimensions. Nevertheless, we have shown above that one can only appreciate the rhythms, periodic and aperiodic, seen in numerical simulations of the one-dimensional cable equation by applying concepts drawn from non-linear mathematics. Remarkably, much of that dynamics can be accounted for by the bifurcation structure of a simple non-linear one-dimensional map. It remains to be seen how these concepts can be extended to the case of propagation of the action potential in the three-dimensional structure of the heart, where reentrant circuits can form.

We thank Chris Henri for help with computer programming, Sandra James and Christine Pamplin for typing the manuscript, and Robert Lamarche for photographing the figures. We also thank Dante Chialvo for providing us with the reference to the work of Hescheler \& Speicher (1989).

Supported by a Vector Computer Access grant from the Natural Sciences and Engineering Research Council of Canada, and an operating grant from the Medical Research Council of 
Canada. Tim Lewis thanks the Fonds de la Recherche en Santé du Québec (FRSQ) and the Fonds pour la Formation de Chercheurs et l'Aide à la Recherche (FCAR) for support.

\section{REFERENCES}

Adam, D. R., Akselrod, S. \& Cohen, R. J. (1982). Estimation of ventricular vulnerability to fibrillation through $\mathrm{T}$-wave time series analysis. In: Computers in Cardiology 1981. pp. 307-310. Silver Spring: IEEE Computer Society.

Adam, D. R., Smith, J. M., Akselrod, S., Nyberg, S., Powell, A. O. \& Cohen, R. J. (1984). Fluctuation in T-wave morphology and susceptibility to ventricular fibrillation. $J$. Electrocardiol. 17 , 209-218.

Arneodo, A., Coullet, D., Tresser, C., Libchaber, A., Maurer, J. \& d'Humiere, D. (1983). On the observation of an uncompleted cascade in a Rayleigh-Bénard experiment. Physica 6D, 385-392.

BeELER, G. W. \& REUTER, H. (1977). Reconstruction of the action potential of ventricular myocardial fibres. J. Physiol., Lond. 268, 177-210.

BÉlAIR, J. \& GLASS, L. (1985). Universality and self-similarity in the bifurcations of circle maps. Physica 14D, 143-154.

BOYETT, M. R. \& JEWELL, B. R. (1978). A study of the factors responsible for rate-dependent shortening of the action potential in mammalian vectricular muscle. $J$. Physiol., Lond. 285, 359-380.

Chialvo, D. R., Gilmour, R. F. JR. \& Jalife, J. (1990). Low dimensional chaos in cardiac tissue. Nature, Lond. 343, 653-657.

Chialvo, D. R. \& JAlife, J. (1987). Non-linear dynamics of cardiac excitation and impulse propagation. Nature, Lond. 330, 749-752.

Clay, J. R., Guevara, M. R. \& Shrier, A. (1984). Phase resetting of the rhythmic activity of embryonic heart cell aggregates. Experiment and theory. Biophys. J. 45, 699-714.

Coste, J. \& Peyraud, N. (1982). A new type of period-doubling bifurcations in one-dimensional transformations with two extrema. Physica 5D, 415-420.

DE BEER, E. L. (1977). Atrioventricular conduction. An experimental and theoretical study of nodal action potentials and propagation times. Doctoral Thesis. Utrecht: University of Utrecht.

DilLY, S. G. \& LAB, M. J. (1988). Electrophysiological alternans and restitution during acute regional ischaemia in myocardium of anaesthetized pig. J. Physiol., Lond. 402, 315-333.

Drouhard, J.-P. \& RoBerge, F. A. (1982). A simulation study of the ventricular myocardial action potential. IEEE Trans. Biomed. Eng. BME-29, 494-502.

Dubois, M., Rubio, M. A. \& Berge, P. (1983). Experimental evidence of intermittencies associated with a subharmonic bifurcation. Phys. Rev. Lett. 51, 1446-1449.

ElHaRRAR, V. \& SURAWICZ, B. (1983). Cycle length effect on restitution of action potential duration in dog cardiac fibers. Am. J. Physiol. 244, H782-H792.

Feingold, M., Gonzalez, D. L., Piro, O. \& Viturro, H. (1988). Phase locking, period doubling, and chaotic phenomena in externally driven excitable systems. Phys. Rev. 37A, 4060-4063.

Folas, C., SELl, G. R. \& TEMAM, R. (1988). Inertial manifolds for nonlinear evolutionary equations. J. Diff. Eqns. 73, 309-353.

GetTES, L. S. \& REUTER, H. (1974). Slow recovery from inactivation of inward currents in mammalian myocardial fibres. J. Physiol., Lond. 240, 703-724.

Glass, L., Guevara, M. R., Belair, J. \& Shrier, A. (1984). Global bifurcations of a periodically forced biological oscillator. Phys. Rev. 29A, 1348-1357.

Goldberger, A. L., Shabetai, R., Bhargava, V., West, B. J. \& Mandell, A. J. (1984). Nonlinear dynamics, electrical alternans, and pericardial tamponade (Editorial). Am. Heart J. 107, 1297-1299.

Grebogi, C., OTt, E., PelikAN, S. \& Yorke, J. A. (1984). Strange attractors that are not chaotic. Physica 12D, 261-268.

GreboGi, C., OTT, E. \& YORKE, J. A. (1983). Crises, sudden changes in chaotic attractors, and transient chaos. Physica 7D, 181-200.

GuevarA, M. R. (1984). Chaotic cardiac dynamics. Doctoral Thesis. Montreal: McGıl University.

Guevara, M. R. (1988). Spatiotemporal patterns of block in an ionic model of cardiac Purkinje fibre. In: From Chemical to Biological Organization (Markus, M., Müller, S. C. \& Nicolis, G., eds) pp. 273-281. Berlin: Springer.

GueVARA, M. R. (1990). Iteration of the human atrioventricular (AV) recovery curve predicts many rhythms of AV block. In: Theory of Heart (Glass, L., Hunter, P. \& McCulloch, A., eds) New York: Springer, in press. 
Guevara, M. R. \& Glass, L. (1982). Phase locking, period doubling bifurcations and chaos in a mathematical model of a periodically driven oscillator: a theory for the entrainment of biological oscillators and the generation of cardiac dysrhythmias. $J$. math. Biol. 14, 1-23.

GuevarA, M. R. \& SHRIER, A. (1990). Rhythms produced by high-amplitude periodic stimulation of spontaneously beating aggregates of embryonic chick ventricular myocytes. Ann. N.Y. Acad. Sci. 591, 11-22.

Guevara, M. R., Glass, L. \& Shrier, A. (1981). Phase locking, period doubling bifurcations, and irregular dynamics in periodically stimulated cardiac cells. Science 214, 1350-1353.

Guevara, M. R., Alonso, F., Jeandupeux, D. \& van Ginneken, A. C. G. (1989). Alternans in isolated ventricular myocytes. In: Cell to Cell Signalling: From Experiments to Theoretical Models (Goldbeter, A., ed.) pp. 551-563. London: Academic Press.

GuevarA, M. R., Shrier, A. \& Glass, L. (1986). Phase resetting of spontaneously beating embryonic ventricular heart cell aggregates. Am. J. Physiol. 251, H1298-H1305.

Guevara, M. R., Ward, G. Shrier, A. \& Glass, L. (1984). Electrical alternans and period doubling bifurcations. In: Computers in Cardiology 1984. pp. 167-170. Silver Spring: IEEE Computer Society.

Guevara, M. R., Shrier, A. \& Glass, L. (1990). Chaotic and complex cardiac rhythms. In: Cardiac Electrophysiology: From Cell to Bedside ((Zipes, D. P. \& Jalife, J., eds) Philadelphia: Saunders, in press.

Hashimoto, H., SUzUKi, K. \& NAKAShimA, M. (1984). Effects of the ventricular premature beat on the alternation of the repolarization phase in ischemic myocardium during acute coronary occlusion in dogs. $J$. Electrocardiol. 17, 229-238.

Hescheler, J. \& SPEICHER, R. (1989). Regular and chaotic behaviour of cardiac cells stimulated at frequencies between 2 and $20 \mathrm{~Hz}$. Eur. Biophys. J. 17, 273-280.

HiNES, M. (1984). Efficient computation of branched nerve equations. Int. J. Bio-Med. Comput. 15, 69-76.

HoffMAN, B. F. \& SUCKLING, E. E. (1954). Effect of heart rate on cardiac membrane potentials and the unipolar electrogram. Am. J. Physiol. 179, 123-130.

Jensen, J. H., Christiansen, P. L. \& Scott, A. C. (1984). Chaos in the Beeler-Reuter system for the action potential of ventricular myocardial fibers. Physica 13D, 269-277.

KAhLert, C. \& Rössler, O. E. (1984). Chaos as a limit in a boundary value problem. $Z$. Naturforsch. 39a, 1200-1203.

KAPLAN, D. T. (1989). The dynamics of the cardiac electrical instability. Doctoral Thesis. Cambridge: MIT.

MAHLER, Y. \& RoGel, S. (1970). Interrelation between restitution time-constant and alternating mechanical contractility in dogs. Clin. Sci. 39, 625-639.

MALlet-PARET, J. \& NuSSBAUM, R. D. (1986). A bifurcation gap for a singularly perturbed delay equation. In: Chaotic Dynamics and Fractals (Barnsley, M. F. \& Demko, S. G., eds) pp. 263-286. S. Diego: Academic Press.

Matsumoto, G., Aimara, K. Hanyu, Y., Takahashi, N., Yoshizawa, S. \& Nagumo, J. (1987a). Chaos and phase locking in normal squid axons. Phys. Lett. A 123, 162-166.

Matsumoto, G., Takahashi, N. \& Hanya, Y. (1987b). Chaos, phase locking and bifurcation in normal squid axons. In: Chaos in Biological Systems (Degn, H., Holden, A. V. \& Olsen, L. F., eds) pp. 143-156. New York: Plenum.

MAY, R. (1976). Simple mathematical models with very complex dynamics. Narure, Lond. 261, 459-467.

MAYER-KRESS, G. \& HAKEN, H. (1982). Transition to chaos for maps with positive Schwarzian derivative. In: Evolution of Order and Chaos (Haken, H., ed.) pp. 183-186. Berlin: Springer.

Miller, R. N. \& Rinzel, J. (1981). The dependence of impulse propagation speed on firing frequency, dispersion, for the Hodgkin-Huxley model. Biophys. J. 34, 227-259.

MiNES, G. R. (1913). On dynamic equilibrium in the heart. J. Physiol, Lond. 46, 349-383.

Moe, G. K., Jalife, J., Mueller, W. J. \& MOE, B. (1977). A mathematical model of parasystole and its application to clinical arrhythmias. Circulation 56, 968-979.

Moore, D. R., ToOmRe, J., KNOBloch, E. \& WeIss, N. O. (1983). Period doubling and chaos in partial differential equations for thermosolutal convection. Nature, Lond. 303, 663-667.

NolAsCo, J. B. \& DAHLEN, R. W. (1968). A graphic method for the study of alternation in cardiac action potentials. J. appl. Physiol. 25, 192-196.

Pomeau, Y. \& MANNeville, P. (1980). Intermittent transition to turbulence in dissipative dynamical systems. Commun. math. Phys. 74, 189-197.

Robinson, R. B., Boyden, P. A., Hoffman, B. F. \& Hewlett, R. W. (1987). Electrical restitution process in dispersed canine Purkinje and ventricular cells. Am. J. Physiol. 253, H1018-H1025.

Savino, G. V. \& VAlentinuzzi, M. E. (1988). Ventricular fibrillation-defibrillation in the toad Bufo paranemis. Int. J. Cardiol. 19, 19-25.

Savino, G. V., Romanelli, L., Gonzalez, D. L., Piro, O. \& Vantinuzzi, M. E. (1989). Evidence for chaotic behavior in driven ventricles. Biophys. J. 56, 273-280. 
SCoTT, S. W. (1979). Stimulation simulations of young yet cultured beating heats. Doctoral Thesis. Buffalo: State University of New York.

SHARP, G. H. \& JOYNER, R. W. (1980). Simulated propagation of the cardiac action potentials. Biophys. J. 31, 403-423.

Shrier, A., Dubarsky, H., Rosengarten, M., Guevara, M. R., Nattel, S. \& Glass, L. (1987). Prediction of complex atrioventricular rhythms in humans with use of the atrioventricular recovery curve. Circulation 76, 1196-1205.

Smith, J. M, Clancy, E. A., Valeri, C. R., Ruskin, J. N. \& Cohen, R. J. (1988). Electrical alternans and cardiac electrical instability. Circulation 77, 110-121.

Temam, R. (1988). Infinite Dimensional Dynamical Systems in Mechanics and Physics. Berlin: Springer.

ThompSON, J. M. T. \& STEWART, H. B. (1986). Nonlinear Dynamics and Chaos. Geometrical Methods for Engineers and Scientists. Chichester: Wiley.

Tresser, C., Coullet, P. \& ArNeOdo, A. (1980). On the existence of hysteresis in a transition to chaos after a single bifurcation. J. Phys. Lett., Paris 41, L243-L246.

Victorri, B., Roberge, F. A., Boucher, L., Vinet, A. \& Drouhard, J. P. (1984). A simulation study of discontinuous propagation in cardiac muscle. In: Computers in Cardiology 1984. pp. 431-434. Silver Spring: IEEE Computer Society.

Victorri, B., Vinet, A., Roberge, F. A. \& Drouhard, J.-P. (1985). Numerical integration in the reconstruction of cardiac action potentials using Hodgkin-Huxley-type models. Comp. Biomed. Res. 18, 10-23.

Vinet, A., Chialvo, D., Michaels, D. C. \& Jalife, J. (1990). Non-linear dynamics and ionic mechanisms of excitation patterns in models of cardiac myocytes. In: Nonlinear Wave Processes in Excitable Media (Holden, A. V., Markus, M. \& Othmer, H. G., eds) London: Plenum Press, in press. 